\title{
Dictynna
}

Dictynna

Revue de poétique latine

1 | 2004

Varia

\section{La strategia del ragno, ovvero la rivincita di Aracne. Fortuna tardo-antica (Sidonio Apollinare, Claudiano) di un mito ovidiano}

\section{Gianpiero Rosati}

\author{
(2) OpenEdition \\ Journals \\ Edizione digitale \\ URL: http://journals.openedition.org/dictynna/174 \\ DOI: 10.4000/dictynna.174 \\ ISSN: $1765-3142$ \\ Edizione cartacea \\ ISBN: 2-84467-088-1
}

Notizia bibliografica digitale

Gianpiero Rosati, « La strategia del ragno, ovvero la rivincita di Aracne. Fortuna tardo-antica (Sidonio Apollinare, Claudiano) di un mito ovidiano », Dictynna [En ligne], 1 | 2004, mis en ligne le 15 novembre 2010, consulté le 10 septembre 2020. URL : http://journals.openedition.org/dictynna/174 ; DOI : https://doi.org/10.4000/dictynna.174

Questo documento è stato generato automaticamente il 10 settembre 2020.

\section{(c) (i) (9)}

Les contenus des la revue Dictynna sont mis à disposition selon les termes de la Licence Creative Commons Attribution - Pas d'Utilisation Commerciale - Pas de Modification 4.0 International. 


\title{
La strategia del ragno, ovvero la rivincita di Aracne. Fortuna tardo- antica (Sidonio Apollinare, Claudiano) di un mito ovidiano
}

\author{
Gianpiero Rosati
}

Sperduto in questo tessuto - questa tessitura - il

soggetto vi si disfa simile a un ragno che si dissolva da sé nelle secrezioni costruttive della sua tela.

R. Barthes

\section{1) Araneola, un'Aracne più conciliante}

1 Fra i molti personaggi-simbolo che Ovidio ha consegnato alla cultura europea, c'è anche quello di Aracne, l'abilissima tessitrice di cui Minerva punisce l'insolente superbia trasformandola in ragno. La storia è narrata nel sesto libro delle Metamorfosi (1-145) : ammirata da tutti per la sua perizia, Aracne nel suo orgoglio non si riconosce inferiore nemmeno a Minerva, la dea tutelare di quest'arte, e respinge i consigli di moderazione : la sfida anzi a una gara di tessitura in cui le due rivali tessono due arazzi, ispirati da principî etici ed estetici opposti (senso della gerarchia, dell'ordine classico, del decoro nell'arazzo di Minerva; anticonformismo, denuncia del pre-potere degli dei, fluidità ellenistica delle forme in quello di Aracne). La stessa Minerva è costretta ad ammettere l'eccellenza dell'opera della rivale, che dal confronto non esce quindi sconfitta, ma ciò nonostante (o proprio per questo) la dea beffardamente la punisce con una metamorfosi in ragno, condannandola così a tessere per sempre, in perenne ricordo della sua sconfitta.

2 Aracne entra quindi nella tradizione letteraria come emblema dell'arte raffinata della tessitura (Penelope melius, levius torquetis Arachne suona un verso di Giovenale, 2.56) e lo 
resterà ben oltre i confini dell'antichità (cfr. ad es. Ariosto, Orlando 7.23 Ruggiero entrò ne' profumati lini / che pareano di man d'Aracne usciti). Ma quello della tessitura è notoriamente un campo semantico che ha implicazioni culturali di grande rilievo nella cultura occidentale,${ }^{1} \mathrm{e}$ in modo particolare l'arte meticolosa e raffinata del tessere è tradizionalmente assimilata a quella del comporre poesia; per cui ad Aracne tocca il destino di essere anche figura mitico-narrativa della metafora di 'testo'. Una metafora per noi così diffusa e familiare da non esser più percepita come tale (e perciò morta, lessicalizzata) : $:^{2}$ la storia ovidiana di Aracne non solo ne è l'illustrazione narrativa più compiuta, ma costituisce una sorta di aition della metafora stessa (e di quella associata del poeta-ragno). ${ }^{3} \mathrm{Al}$ tempo stesso, quella storia è anche un apologo sui rapporti fra arte e potere, sulla brutalità con cui il potere mortifica le ambizioni di autonomia dell'artista ; e Aracne diventa così figura dell'artista orgoglioso e consapevole, un ruolo che riaffiorerà in varie fasi della cultura europea $\mathrm{e}$ in artisti così diversi come Dante ${ }^{4} \mathrm{o}$ Velázquez. ${ }^{5}$

3 Non stupisce dunque che il personaggio di Aracne susciti una nuova e particolare attenzione fra i letterati della tarda antichità e dell'alto medioevo, quando la ricercatezza dell'elaborazione poetica, e la consapevolezza che la sostiene, raggiungono livelli davvero molto avanzati. Vediamo ad es. che il personaggio di Aracne come figura del poeta raffinato tessitore di ricami verbali sarà presente all'autocoscienza letteraria di un poeta del VI sec., che si mostra erede consapevole dell'intera tradizione latina ("l'ultimo poeta romano», secondo la nota definizione di F. Leo), come Venanzio Fortunato, autore fra l'altro di quei veri arazzi di parole che sono i carmi figurati (per cui egli richiama espressamente la tecnica del ragno: quod velut aragnaea arte videmur picta fila miscere, carm. 5.6 praef. 16). Ed è proprio su un paio di episodi interessanti della fortuna tardo-antica di Aracne che qui vorrei soffermarmi, cominciando da un componimento di Sidonio Apollinare, il poeta funzionario imperiale, e poi vescovo di Clermont-Ferrand, che negli ultimi anni dell'impero ormai agonizzante, di fronte all'incalzare dei 'barbari', vede nell'attività letteraria in forme classiche una difesa della propria identità romana. ${ }^{6}$

4 Uno dei suoi due epitalami, il carmen 15 delle nostre edizioni (l'altro è il carm. 11), composto da 201 esametri e introdotto da una prefazione di 30 endecasillabi falecii, preceduta a sua volta da una lettera in prosa indirizzata al dedicatario, celebra le nozze della nobile Araneola con Polemio, un aristocratico impegnato nell'attività politica (sarà praefectus praetorio Galliarum alcuni anni più tardi) e protagonista nei circoli di cultura nella Gallia del quinto secolo (oltre a vantare una discendenza da Tacito, ha forti interessi per la filosofia neoplatonica). ${ }^{7}$

5 Il fatto che tra i nomi di Arachne e di Araneola intercorrano differenze prosodiche (come la diversa quantità delle prime due sillabe, verificabile, per il secondo idionimo, nell'unica sua occorrenza metrica, a incipit dell'esametro 147 di Sidonio) ${ }^{8}$ non è un ostacolo a mettere in relazione con il personaggio ovidiano l'Araneola di Sidonio. L'idionimo Araneola, mai attestato altrove ${ }^{9}$ (mentre come nome comune ricorre in Cicerone, nat. deor. 2.123 in araneolis aliae... aliae...), ${ }^{10}$ è ovviamente diminutivo di aranea, con spiccata funzione ipocoristico-affettiva, che avrà voluto compendiare in emblema la quintessenza delle virtù femminili : $:^{11}$ un nome insomma, "ragnetto", che nelle intenzioni di chi lo aveva assegnato era già un elogio, e che il poeta 'non poteva', pur a prezzo di forzature metriche, non far entrare nel testo. D'altra parte la connessione anche linguistica fra Arachne e aranea è indubbio che sia istituita dallo stesso Ovidio, il 
quale, chiudendo l'episodio delle Metamorfosi, fornisce sia l'aition del ragno sia un rapporto di continuità appunto anche linguistica fra il nome latino dell'insetto e quello greco (6.145 antiquas exercet aranea telas); e del resto altre analoghe anomalie linguistiche non impediscono oggi agli studiosi di ricondurre l'etimo del latino aranea al gr. ’’ $\rho \alpha ́ x v \eta .{ }^{12}$

6 Ma oltre a non esservi elementi impedienti, vedremo che sono numerosi e cospicui gli argomenti che inducono a vedere dietro il personaggio di Araneola, e dell'epitalamio che la celebra, l'ipotesto ovidiano di Aracne e della sua gara con Minerva, ${ }^{13}$ un ipotesto anzi che è un presupposto necessario per la piena interpretazione di un epitalamio che esige un lettore in grado di accompagnare Sidonio nei percorsi della sua densa tramatura intertestuale. Il destino letterario della giovane sposa Araneola era insomma già iscritto nel suo nome. E trovandosi a dover celebrare un nome appunto così carico di memoria poetica, non si può pensare che il letteratissimo Sidonio, pronto a scherzare sulle implicazioni 'colte' anche del proprio (carm. 13.26 ss. hoc te Sidonius tuus precatur : / sic te Sidonio recocta fuco / multos purpura vestiat per annos), ${ }^{14}$ perdesse l'occasione di mettere a frutto la circostanza per valorizzare tutte le potenzialità letterarie che quel nome in nuce condensava. A tale scopo doveva ovviamente adattare la circostanza dell'obbligo celebrativo alle peculiarità del personaggio ovidiano e della sua caratterizzazione : il nome di Araneola rendeva insomma inevitabile l'associazione, se non proprio l'identificazione, con Aracne, ma questi non era un personaggio da accostare o sovrapporre alla dedicataria di un epitalamio se non dopo qualche accorgimento.

7 Alla memoria letteraria del pubblico colto cui Sidonio si rivolge Aracne presentava infatti i tratti di una donna empia e ribelle all'autorità divina, che con gesto di hybris aveva sfidato Minerva nell'arte di cui la dea stessa è inventrice e nume tutelare; nella gara, per di più, aveva composto un'opera figurata in cui aveva irriso la maestà divina rappresentando un catalogo di disonorevoli avventure erotiche degli dei più famosi, a iniziare da Giove, a danno di indifese fanciulle mortali. Un atteggiamento così audace $\mathrm{e}$ oltraggioso da provocare appunto la dura reazione di Minerva e la condanna alla metamorfosi in ragno. Per poter dunque celebrare Araneola accostandola ad Aracne andavano neutralizzati, o comunque attenuati con l'ironia, i tratti anti-teologici ed eversivi : un atteggiamento di aperta ribellione all'autorità costituita non era la premessa migliore per onorare la sposa di un esponente di spicco dell'establishment politico e sociale della Gallia come Polemio.

D'altro lato bisognava riscoprire altri aspetti, altre caratteristiche della figura di Aracne, e dell'animale che dalla sua metamorfosi era nato, il ragno, per esaltarne le qualità etico-ideologiche positive, edificanti, di emblema delle virtù femminili. Ed è appunto questa complessa operazione che Sidonio realizza. Egli recupera dunque il filo di quella tradizione che identificava nell'arte del ragno l'emblema della pazienza e della meticolosità (Front. Laudes neglegentiae, p. 219, 6-8 v.d.H. texendi vero araneas diligentiores esse quam Penelopam ullam vel Andromacham), facendone l'arte forse più docta e ammirata nell'intera sfera animale (Sen. epist. 121.22-3). ${ }^{15}$ Era stato il ragno che, secondo Democrito (frg. $154 \mathrm{D}-\mathrm{K}$ ), aveva insegnato all'umanità l'arte della tessitura, e secondo Plutarco la tela che esso tesse, ammirevole per la finezza del filo, è un modello di

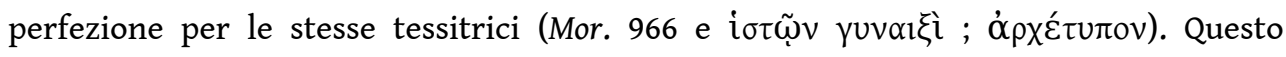

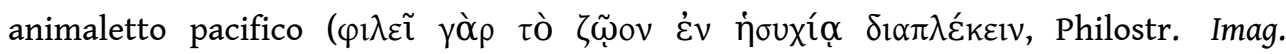
2.28.24-5), che tesse la sua tela raffinata in luoghi tranquilli e appartati (Ov. am. 1.14.8 
deserta sub trabe), poteva insomma prestarsi a diventare un perfetto simbolo delle virtù che la cultura antica associava stabilmente alla donna, un modello di laboriosità e di vita appartata nel chiuso spazio domestico.

Mentre Araneola, che nel suo nome ha un destino, è legata alla sfera femminile e alle attività domestiche (la donna-ragno che svolge il suo lavoro silenzioso nel chiuso della casa), Polemio è un filosofo, un cultore della sapienza, oltre ad avere un nome che lo associa alla guerra, un'attività che viceversa è eminentemente maschile e pubblica (l'opposizione tra fare la guerra e filare-tessere è nitidamente fissata già nel sesto dell' Iliade). E come Minerva è la divinità tutelare delle attività femminili, allo stesso modo è dea della sapienza nonché della guerra: questa dea dall'appartenenza sessuale notoriamente problematica, è da un lato la divinità dei lavori femminili, in primo luogo la filatura-tessitura, ma al tempo stesso è la belli metuenda virago (Ov. met. 2.765), la dea virile per antonomasia e amante delle armi. ${ }^{16}$ Il ruolo che Minerva viene quindi ad assumere all'interno dell'epitalamio è assolutamente centrale, tanto da assurgere a dea pronuba, un ruolo che nella tradizione dell'epitalamio compete regolarmente a Venere. La dea dell'eros viene dunque sostituita nelle sue funzioni dalla dea che all'eros è più estranea e refrattaria (casta per antonomasia, assume spesso il ruolo di vendicatrice di vergini, come ci ricorda il v. 2 Phoebados Iliacae raptum satis ulta pudorem); un'inversione a cui sembra corrispondere uno scambio delle parti fra gli sposi (che ha fatto più volte parlare di parodia) $:{ }^{17}$ qui è infatti lo sposo che, di fronte alle nozze, trattenuto da scrupoli filosofici si mostra timido e pudibondo (192 haerentem ; 196 vultu... modesto), mentre la sposa vedremo che esibisce un atteggiamento più libero e disinvolto (come rileverà la stessa Minerva a 186).

La scena dell'epitalamio è Atene, la città che da Atena-Minerva prende fin il nome : lì essa sta tornando da un'azione di guerra (la distruzione della flotta dei Greci reduci da Troia in quanto colpevoli del ratto di Cassandra nel tempio della dea : siamo quindi poco dopo la fine della guerra) con indosso la pesante armatura (di cui viene fornita una dettagliata descrizione, con particolare insistenza sulle scene della Gigantomachia raffigurate sullo scudo: 4-33) e tutti gli attributi militari. Ad Atene si trovano due templi, il primo dei quali dedicato alla filosofia; e di qui inizia un catalogo di filosofi (a partire dai Sette Sapienti), e l'illustrazione dei rispettivi campi speculativi, che culmina in Platone e nella sua scuola (36-117) :

Hic duo templa micant, quorum supereminet unus

ut meritis sic sede locus, qui continet alta

scrutantes ratione viros...

11 In questo tempio, accanto al suo Platone, vive Polemio sotto la protezione della stessa Sapientia (118-25). A bilanciare l'ampia digressione sul tempio della filosofia, emblema del mondo (ovviamente maschile : cfr. 38 viros) di Polemio e dei suoi interessi, segue un'analoga rappresentazione dell'altro tempio, il textrinum Minervae, e delle attività femminili che vi si svolgono (126 ss.). Ne risulta una struttura bilanciata che giustappone i due mondi, rispettivamente dello sposo e della sposa, ${ }^{18}$ tutti e due peraltro uniti dalla comune protezione di Minerva, dea della sapienza non meno che delle arti femminili, il cui ampio spazio nell'introduzione dell'epitalamio (1-35) funge appunto da elemento unificante nell'economia generale del componimento.

La presentazione del laboratorio di Minerva è introdotta dalla accurata descrizione di tre preziosi manufatti tessili. ${ }^{19}$ Sui mantelli di Giove (127-31) e di Nettuno (132-4) ${ }^{20}$ sono effigiati i simboli delle rispettive sfere di potere (il fulmine per il dio del cielo, la 
tempesta per quello del mare); mentre più complessa è l'ekphrasis della terza vestis, quella di Ercole (135 ss.). A una prima parte (136-9), che illustra analiticamente la rappresentazione dell'impresa dell'uccisione dei serpenti, fa seguito un secco catalogo (141-3) che in lunga serie asindetica elenca singoli nomi o attributi come marchi di riconoscimento delle varie imprese (che si presume quindi fossero tutte ricamate sul tessuto). ${ }^{21}$

Una formula riassuntiva indica come autrici di questi e di simili manufatti le fanciulle che lavorano nel textrinum; ma su questo sfondo generico si staglia la figura della protagonista :

Hoc opus et si quid superest, quod numina vestit, virgineae posuere manus. Sed in agmine toto inter Cecropias Ephyreiadasque puellas Araneola micat ; proprias conferre laborat ipsa Minerva manus, calathisque evicta recedens cum tenet haec telas vult haec plus tela tenere (144-9)

La scena 'di genere', cioè l'agmen delle ancelle (in qualche modo 'mitizzate' dagli epiteti, ${ }^{22}$ oltre che dal fatto di tessere vesti divine) che filano-tessono attorno alla loro domina, è appunto funzionale a far risaltare l'entrata in scena di Araneola (che micat come Minerva al suo primo apparire al v. 4). Accanto a lei (finora mai menzionata, nemmeno indirettamente) è raffigurata Minerva che cerca la competizione (laborat) diretta con lei e poi, evidentemente riconoscendosi sconfitta (evicta), si allontana dal 'campo di battaglia' (quello dei calathi, i cestelli di lana, in cui avviene il confronto). L'enunciazione del nome Araneola comporta cioè, automaticamente, come per un riflesso condizionato nella memoria del lettore colto, il ricordo della mitica Aracne e della sfida fatale lanciata alla dea. Appena evocato, però, il modello letterario viene subito corretto : diversamente da Ovidio, dov'era Aracne a cercare provocatoriamente la sfida (certet... mecum, 6.25 ; cur haec certamina vitat ?, 42), qui è Minerva stessa che vuole (laborat) il confronto e che subito si riconosce sconfitta, ritirandosi per tornare a occuparsi piuttosto della sfera dei tela, del mondo 'maschile' delle armi e della guerra. L'opposizione che struttura il v. 149 (tenet haec telas... haec... tela tenere) riflette la distinzione di campo fra le due figure, ognuna titolare di una sfera di eccellenza (cui corrispondono rispettivamente i due poli della paronomasia telae/tela). L'immediata correzione del modello evocato serve a neutralizzare la tipologia negativa del personaggio, quella che il lettore conosce; e al tempo stesso, rovesciando la situazione e attribuendo a Minerva lo spontaneo riconoscimento del primato di Araneola nell'arte tessile, sfrutta l' iperbole encomiastica per pagare l'inevitabile omaggio alla protagonista e dedicataria dell'epitalamio.

Mentre la presenza di Polemio nel tempio della filosofia non evoca alcuna idea di competizione, e tanto meno mette in discussione il primato 'divino' di Sapientia (che si prende cura di lui : $118 \mathrm{~s}$.) o anche del summus Platone (99; detto anche magister a 192), non così accade per Araneola, che la dea stessa riconosce superiore sul suo stesso terreno, cioè nel campo dell'arte tessile. A dimostrazione di questa eccellenza artistica vengono citati dei drappi tessuti da Araneola per il padre Magno, console designato: una toga consolare (150-3), delle clàmidi (154-7) e una trabea, la cui decorazione viene poi descritta in dettaglio :

Attamen in trabea segmento luserat alto

quod priscis inlustre toris. Ithacesia primum 
fabula Dulichiique lares formantur et ipsam

Penelopam tardas texit distexere telas (158-61).

Il soggetto della decorazione è il più antico mito letterario di fedeltà coniugale, quello di Penelope, individuato attraverso gli elementi simbolici del luogo, la casa (lares), e dell'azione di lei, che dis-tesse di notte la tela tessuta di giorno (rinviando così sine die la scadenza delle nuove nozze volute dai proci). Ad esso seguono gli exempla di Orfeo (162-4), di Alcesti (165-7) e di Ipermestra (168-73), tutti intesi a celebrare appunto l'amore coniugale, un tema quanto mai appropriato in un epitalamio ${ }^{23}$ (che poi la trabea fosse destinata non allo sposo, ma al padre futuro console, è un particolare su cui qui sorvoliamo).

Assai meno opportuni in questo contesto nuziale, e contraddittori con i soggetti precedenti, sono quelli che Araneola passa a rappresentare successivamente (174-6) :

Iamque Iovem in formas mutat quibus ille tenere

Mnemosynam, Europam, Semelen, Ledam, Cynosuram

serpens, bos, fulmen, cygnus, Dictynna solebat.

metamorfosi assunte da Giove per le sue avventure erotiche (a ognuna delle cinque 'vittime' elencate per nome nel primo verso corrisponde nel secondo la forma assunta dal dio per attuare l'inganno) ; un soggetto non certo appropriato per una novella sposa, e soprattutto lo stesso soggetto scabroso con cui Aracne, nell'ipotesto ovidiano, aveva irriso la pretesa maestà degli dei suscitando l'ira di Minerva : ${ }^{24}$

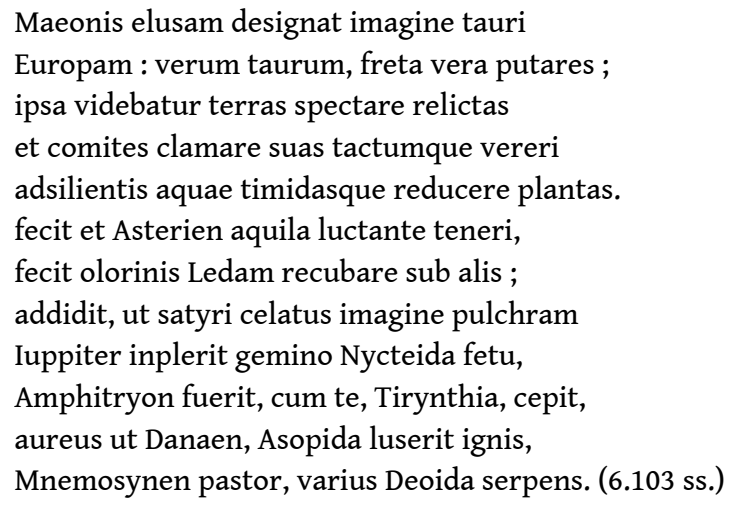

Tre delle cinque vicende amorose di Giove (oltre a quella di Danae, cui si accennerà subito dopo) sono comprese nell'insolente catalogo di Aracne, mentre le altre due, Semele e Callisto, erano narrate nei libri precedenti del poema ovidiano, rispettivamente il terzo e il secondo. ${ }^{25}$ Ad ogni modo colpisce, dopo un soggetto edificante come la devozione coniugale, il brusco passaggio a una tematica così diversa e soprattutto così poco rispettosa della divina presenza di Minerva (una divinità che per di più, come abbiamo detto, è notoriamente allergica al sesso). Lo conferma l'imbarazzo, se non il fastidio, che la dea manifesta di fronte a questi nuovi soggetti distogliendo lo sguardo dallo ‘spettacolo' di Araneola al telaio per volgerlo altrove :

Iamque opus in turrem Danaae pluviamque metalli

ibat et hic alio stillabat Iuppiter auro,

cum virgo aspiciens vidit Tritonida verso

lumine doctisonas spectare libentius artes;

commutat commota manus ac pollice docto ${ }^{26}$

pingere philosophi victricem Laida coepit,

quae Cynici per menta feri rugosaque colla

rupit odoratam redolenti forpice barbam (177-84). 
A trattenere Araneola dal continuare a tessere il suo catalogo figurativo alla maniera di Aracne è appunto il gesto di Minerva che preferisce guardare in altra direzione (probabilmente verso l'altro tempio, quello dei filosofi) ${ }^{27}$ esprimendo il disagio per quei soggetti sconvenienti. Araneola, turbata, cambia allora nuovamente soggetto e rappresenta la vittoria di Laide (la cortigiana per antonomasia, cioè un emblema dell'eros) su Diogene (a sua volta emblema della superiorità della sapientia sulle passioni terrene) : ${ }^{28}$ ripiega cioè su un soggetto meno imbarazzante, ma non rinuncia a un atteggiamento di ironia nei confronti della dea. Il taglio, da parte dell'etera, della barba maleodorante del filosofo più di ogni altro ferus, ascetico e intransigente, « rappresenta il momento in cui l'inflessibilità del filosofo cede alle lusinghe amorose " ${ }^{29} \mathrm{e}$ dunque simboleggia la vittoria dell'eros sulla presunta superiorità della filosofia.

21 Il nuovo soggetto rappresenta cioè una versione attenuata, trasferita sul piano terreno e umano, dello stesso tema di Aracne, cioè del 'trionfo di amore' su chi (come gli dei o $\mathrm{i}$ filosofi) si proclama superiore alle passioni umane e invece non sa resistere alla loro seduzione. Per quanto corretto e spogliato dei suoi tratti più aggressivi e blasfemi, il modello di Aracne non viene cioè obliterato del tutto, ma conserva qualcosa dell'antica polemica contro la dea, stemperata qui dall'ironia. Di fronte all'atteggiamento scherzosamente provocatorio, al «piccolo dispetto» (Morelli) di Araneola, Minerva sorride e la ammonisce :

Subrisit Pallas castoque haec addidit ore :

« Non nostra ulterius ridebis dogmata, virgo

philosopho nuptura meo; mage flammea sumens

hoc mater sine texat opus. Consurge sophorum

egregium Polemi decus, ac nunc Stoica tandem

pone supercilia et Cynicos imitatus amantes

incipies iterum parvum mihi ferre Platona » (185-91)

Anche quella di Araneola, come già quella di Aracne, è un'irrisione - seppure garbata $\mathrm{e}$ priva di ogni aggressività - dei dogmata, dei principî della dea che presiede alla sfera della razionalità e della castità, al rifiuto della passione erotica ; una sfida scherzosa da parte di una promessa sposa la quale spera «che le sue grazie non cadano nell'indifferenza di un marito dal fiero cipiglio stoico $» .^{30}$

Ma chi è questa madre tessitrice a cui Minerva, sorridendo bonaria, consiglia di lasciare la tessitura di quei soggetti così inopportuni ? Forse la madre naturale di Araneola, una madre per noi del tutto ignota e cui si accredita un'abilità nell'arte in cui eccelle la figlia. ${ }^{31}$ Ma perché a una tale madre quel tipo di soggetti pittorici dovrebbero essere più confacenti? Forse solo perché a una matrona esperta si può concedere un atteggiamento più disinvolto e meno castigato di quello che si esige da una virgo ? Io credo più probabile un'altra spiegazione, e cioè che quella mater altri non sia che la madre mitico-letteraria di Araneola, vale a dire Aracne, l'empia e insolente tessitrice ovidiana il cui modello Minerva le sconsiglia di imitare. Nella 'piccola Aracne' che è Araneola la dea riconosce cioè la discendente della sua antica rivale, ma la invita ad assumere un atteggiamento diverso e le offre l'occasione di una riconciliazione, appunto le nozze con un suo adepto, il filosofo Polemio.

L'epitalamio di Polemio e Araneola nasconde cioè sotto la veste celebrativa un discorso letterario sul modello che sottostà al personaggio di Sidonio. Del resto non è l'unico indizio di un atteggiamento riflessivo, l'unico gesto di autocoscienza dell'autore che riflette sulla sua attività poetica $; 2$ il che non stupisce certo in un componimento che nel tema della tessitura (a cui appunto la stessa protagonista è così strettamente 
vincolata, fin dal nome) ha il suo nucleo centrale. ${ }^{33}$ Vediamo ad es. il modo in cui viene introdotta Araneola e con lei tutta la sezione sulla tessitura, incluse le diverse ekphraseis :

At parte ex alia textrino prima Minervae palla Iovis rutilat, cuius bis coctus aeno serica Sidonius fucabat stamina murex (126-8)

È difficile resistere alla suggestione che, come già a 13.26 s., quel Sidonius racchiuda un pun su di lui, sul poeta stesso ; che insomma sia lui, Sidonio, il prezioso 'colore' che attraverso la tessitrice Araneola dà la vita ai fili di seta che intrecciano il suo 'testo'.

Ma l'immagine forse più significativa in questo senso dell'intero componimento è quella di Araneola che tesse Penelope, la tessitrice par excellence, nell'atto di dis-tessere la sua tela :

Penelopam tardas texit distexere telas (161).

La particolare intenzione espressiva è rivelata anche da una lieve forzatura della lingua, cioè la coniazione dell'hapax assoluto distexere, che rispetto a retexere (sempre in relazione a Penelope ad es. in Ov. Am. 3.9.30 tardaque nocturno tela retexta dolo e in Cic. Luc. 95 quasi Penelope telam retexens) ha il vantaggio di non prevedere una possibile accezione iterativa (come 'tessere una seconda volta' : cfr. ad es. carm. 9.106), e insiste invece sul sema del dis-fare ciò che è stato fatto, sul percorso inverso a quello creativo. L'immagine di un testo che si forma rappresentandone un altro che si scompone è la metafora più appropriata per una poesia così consapevole della sua natura 'costruita' $\mathrm{e}$ riflessa; e al tempo stesso l'emblema più adeguato anche di un componimento che è appunto costruito sulla decostruzione del suo ipotesto ovidiano.

In questa immagine è insomma condensato il massimo di riflessività di questo testo : una tessitrice, la donna-ragno Araneola, che tesse la tessitrice Penelope (cioè replica il suo proprio modello) intenta a distexere la tela che ha tessuto. C'è un altro testo antico in cui Penelope è coinvolta, attraverso l'immagine del tessere, in un rapporto di analogia metaforica con l'artista che la ritrae. ${ }^{34} \mathrm{Si}$ tratta delle Imagines del greco Filostrato, un autore che Sidonio conosce (lo menziona in epist. 8.3.1), ${ }^{35} \mathrm{e}$ della cui opera sono stati segnalati indizi di un influsso diretto sulle sezioni ecfrastiche di Sidonio. ${ }^{36}$ Nella galleria di Filostrato (2.28) viene descritto un quadro, intitolato 'Telai', in cui il

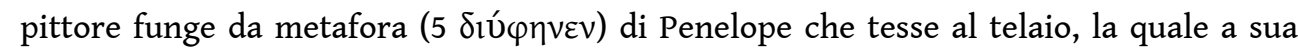
volta è assimilata al ragno (modello-archetipo della tessitura) che accanto a lei fa la sua tela. ${ }^{37}$ Un'evidente insistita rappresentazione figurata della metafora di testo, della tessitura come immagine della creazione artistica, da dove Sidonio potrebbe aver tratto l'idea; anche se in realtà l'immagine e l'intero campo semantico della tessitura conoscono un'amplissima diffusione in tutta la cultura tardo-antica, sia pagana che cristiana. $^{38}$

Esplorando attraverso il personaggio dell'artista-ragno il campo semantico della testualità, il testo di Sidonio riproduce il processo della sua formazione ed enuncia insieme la sua poetica, dichiara la sua natura di poesia elaborata e riflessa. Come già era accaduto in Ovidio e come poi accadrà in altri artisti, anche in Sidonio cioè Aracne sembra fungere da 'figura' dell'artista e della sua autocoscienza, rispecchiare la sua funzione di creatore di un 'testo'. Che è un'altra maniera per Aracne di preservare quella dignità di cui la brutale vendetta della Minerva ovidiana la voleva privare.

Nella sua erede Araneola l'Aracne di Ovidio conosce quindi un riscatto dalla sconfitta e dall'oblio cui era stata condannata. Quest'Araneola non aggressiva e blasfema come 
Aracne, ma ironica e arguta, adotta nei confronti della sua eterna rivale una strategia diversa, non di attacco diretto, violento, ma obliqua, sottilmente ironica, avvolgente, una strategia più adatta a chi vuole misurarsi con un potere superiore. Un atteggiamento conciliante e flessibile le permetterà di riaffermare di fronte alla dea della sapienza la propria verità, e cioè che la ragione, con le sue pretese di superiorità, non è in grado di sottrarsi al potere dell'eros.

\section{2) La vendetta di Aracne}

Un episodio anch'esso significativo del destino di Aracne trasformata in ragno lo troviamo in un altro testo tardo-antico, un poemetto epico di Claudiano anch'esso fortemente segnato dall'influsso delle Metamorfosi ovidiane. Nel primo libro del De raptu Proserpinae viene narrato l'antefatto del ratto vero e proprio (che avrà luogo nel libro successivo) : sotto le pressanti richieste di Plutone che pretende di avere anch'egli una sposa, e minaccia altrimenti di muover guerra ai superi e sovvertire l'ordine cosmico, Giove decide di destinare in nozze al fratello la figlia di Cerere, la giovane Proserpina. Convoca quindi Venere e le affida l'incarico di tramare l'inganno con cui far cadere la fanciulla nella rete del sovrano degli inferi. Accompagnata da Minerva e Diana, Venere si reca in Sicilia, alla reggia di Cerere, dove trova Proserpina intenta a tessere (la scenaemblema della virtù femminile, fissata nella memoria letteraria romana in associazione alla figura di Lucrezia) : sta ricamando un peplo, pensato come dono per la madre (De rapt. $1.246 \mathrm{~s}$.), con scene di soggetto cosmologico (248-70), ${ }^{39} \mathrm{ma}$ l'arrivo improvviso delle dee la induce ad abbandonare il lavoro, che stava completando (269 s.), e che rimane quindi incompiuto : cernit adesse deas imperfectumque laborem / deserit et niveos infecit purpura vultus $(271$ s.). Il mattino successivo Proserpina aderirà all'invito di Venere a recarsi a raccogliere fiori nei prati e lì sarà rapita da Plutone. Quando Cerere, atterrita da presagi funesti, corre a casa in cerca di Proserpina, trova la reggia abbandonata e in preda allo squallore; ma la descrizione della generale desolazione si sofferma soprattutto sugli strumenti dell'arte tessile : semirutas confuso stamine telas / atque interruptas agnoscit pectinis artes (3.155 s.). In particolare, il peplo che Proserpina stava tessendo e aveva dovuto bruscamente interrompere giace nel disordine e sta subendo l'insulto delle ragnatele, il simbolo della trascuratezza e dell'abbandono :40

Divinus perit ille labor, spatiumque relictum

audax sacrilego supplebat aranea textu (157 s.).

31 Stupisce in questa rappresentazione il fatto che, dal momento in cui Proserpina ha interrotto il suo lavoro, sembra sia trascorso non un tempo piuttosto limitato, ma quasi secoli di abbandono e trascuratezza. ${ }^{41}$ Il ragno che tesse la sua tela sul raffinato peplo di Proserpina è come l'emblema conclusivo del generale sovvertimento, che vede le forze brute della natura avere il sopravvento su quel prodotto artistico 'divino'. Il peplo di Proserpina è divinus non solo perché è stato tessuto da una dea, ora per di più sposa del dio sovrano degli inferi, e non solo come epiteto che qualifica l'eccellenza artistica del manufatto, ma anche in un altro senso : perché il soggetto della rappresentazione è una celebrazione dell'ordine cosmico governato dagli dei, è un trionfo della teodicea. ${ }^{42} \mathrm{Il}$ peplo di Proserpina è cioè del tutto simile all'arazzo tessuto da Minerva in Ovidio :43 $\mathrm{di}$ quell'arazzo condivide sia i criteri estetici (armonia e ordine classico) sia quelli ideologici (ammirazione e rispetto dell'autorità degli dei). E quel peplo ora non solo giace nell'incuria e nell'abbandono, ma addirittura perit, va già incontro a una piena (e 
simbolica) distruzione, sancisce la sconfitta di quell'ordine e dell'ideologia che lo sostiene.

L'immagine del ragno che 'completa' la tela incompiuta di Proserpina esprime cioè due idee intimamente contraddittorie : da un lato l'idea della continuità-identità fra il texere della fanciulla e il texere del ragno, ricordando alla memoria mitico-letteraria del lettore l'eziologia che riconduce l'origine del ragno alla vicenda di Aracne, e alla metamorfosi che beffardamente condannava l'abilissima ma empia tessitrice a tessere in eterno sotto forma di ragno. D'altro canto però audax e sacrilego (in forte opposizione a divinus, che rivela in questa chiave tutta la sua funzionalità) smentiscono l'idea di continuità implicita in supplebat, e rievocando la contesa fra l'orgogliosa tessitrice mortale e la divina Minerva ci dicono che la tela tessuta dal ragno è un segno di tutt'altra natura, è il simbolo dell'incuria e della dimenticanza, ${ }^{44}$ della sconfitta dei valori di Minerva (e di Proserpina) e dell'ordine di cui la teodicea dovrebbe essere garante. Quella 'continuità' è insomma tutt'altro che pacifica : la tela che il ragno tesse empiamente sul peplo 'divino', e sull'ideologia che esso celebra, è l'oltraggio postumo che Aracne infligge al suo antico nemico, quasi il corrispettivo dell'offesa di Minerva che aveva distrutto l'arazzo blasfemo della sua rivale (Ov. Met. 6.131 rupit pictas, caelestia crimina, vestes). La forza espressiva di perit ci dice che quel gesto è la vendetta e la rivincita di Aracne su Minerva, la riaffermazione della critica di Aracne al potere stabilito e ai principî morali che esso pretende di avere a fondamento (una critica cui ora proprio il ratto di Plutone ai danni di Proserpina, l'ennesimo esempio di sfrenato erotismo di un dio, offre nuovo alimento). ${ }^{45}$ Nella sua tela Proserpina "reproduit l'harmonie du cosmos que son sacrifice va préserver $"::^{46} \mathrm{ma}$ il prezzo da pagare per la salvaguardia di questo cosmos - vale a dire dei rapporti, e degli equilibri, di potere fra gli dei - va ancora una volta a gravare su una fanciulla innocente che alla logica di quella 'armonia' viene appunto brutalmente immolata ; e l'oltraggio del ragno al 'testo' nel quale la stessa vittima la esaltava artisticamente è l'estrema protesta di chi ha osato denunciare quella logica ed è stato perciò condannato a un'esistenza che è un perenne ricordo della propria sconfitta e umiliazione. ${ }^{47}$

Non è strano che Aracne, la sfortunata artista-ragno condannata a tessere le sue fragili tele, conosca il suo riscatto e una nuova fortuna, dopo l'oblio che ne copre l'esistenza a partire da Ovidio, ${ }^{48}$ in poeti che dell'arte silenziosa del tessere poesia hanno fatto una scelta quasi religiosa. Che poi questa rivincita avvenga in forma criptica e velata si può capire in artisti che da Aracne hanno ereditato la perizia tecnica ma non certo la stessa coraggiosa indipendenza di fronte all'autorità del potere.

\section{BIBLIOGRAFIA}

von Albrecht 1989

M. von Albrecht, Proserpina's tapestry in Claudian's De raptu : tradition and design, « ICS » 14, 1989, 383-90 
von Albrecht 1999

M. von Albrecht, Roman Epic. An Interpretative Introduction, Leiden-Boston-Köln 1999.

Barkan 1986

L. Barkan, The gods made flesh. Metamorphosis \& the pursuit of the paganism, New Haven-

London 1986

Barolini 1987

T. Barolini, Arachne, Argus, and St. John : Transgressive Art in Dante and Ovid, in « Mediaevalia »

$13,1987,207-26$

Bryson 1994

N. Bryson, Philostratus and the imaginary museum, in S. Goldhill-R. Osborne (edd.), Art and text

in ancient greek culture, Cambridge 1994, 255-83

Capponi 1994

F. Capponi, Entomologia pliniana (N.H.XI, 1-120), Genova 1994

Charlet 1991

Claudien, Le rapt de Proserpine, texte ét. et trad. par J.-L. Charlet, Paris 1991

Colton 2000

R.E. Colton, Some Literary Influences on Sidonius Apollinaris, Amsterdam 2000

Consolino 1974

F.E. Consolino, Codice retorico e manierismo stilistico nella poetica di Sidonio Apollinare, in «

ASNP » s. III, 4, 1974, 423-60

Ernout-Meillet 1959

A. Ernout - A. Meillet, Dictionnaire étymologique de la langue latine. Histoire des mots, Paris 19594

Gorni 1979

G. Gorni, La metafora di testo, in « Strum. crit. » 38, 1979, 18-32 (= Metrica e analisi letteraria, Bologna 1993, 137-52)

Gruzelier 1993

Claudian, De raptu Proserpinae, ed. with transl., introd. and comm. by C. Gruzelier, Oxford 1993

Gualandri 1979

I. Gualandri, Furtiva lectio. Studi su Sidonio Apollinare, Milano 1979

Kaufmann 1995

F.-M. Kaufmann, Studien zu Sidonius Apollinaris, Frankfurt a.M.-Berlin-Bern-New York-Paris-Wien 1995

Kellner 1997

Th. Kellner, Die Göttergestalten in Claudians De Raptu Proserpinae, Stuttgart-Leipzig 1997

La Penna 1995

A. La Penna, Gli svaghi letterari della nobiltà gallica nella tarda antichità. Il caso di Sidonio Apollinare, in « Maia » 47, 1995, 3-34

Loyen 1943

A. Loyen, Sidoine Apollinaire et l'esprit précieux en Gaule aux derniers jours de l'empire, Paris 1943

Loyen 1960

Sidoine Apollinaire. Tome I Poèmes, texte établi et trad. par A. Loyen, Paris 1960 
Loyen 1964

A. Loyen, Études sur Sidoine Apollinaire, in « REL » 46, 1968, 83-90

Mesturini 1982

A.M. Mesturini, Due asterischi su Sidonio, in « Sandalion » 5, 1982, 263-76

Morelli 1910

C. Morelli, L'epitalamio nella tarda poesia latina, in « SIFC» $18,1910,319-432$

Pease 1955-58

M. Tulli Ciceronis De natura deorum, ed. by A.S. Pease, Cambridge, Mass. 1955-58

Purgold 1878

K. Purgold, Archäologische Bemerkungen zu Claudian und Sidonius, Gotha 1878

Ravenna 1990

Le nozze di Polemio e Araneola (Sidonio Apollinare, Carmina XIV-XV), introd., testo crit. e comm. a c. di G. Ravenna, Bologna 1990

Roberts 1989a

M. Roberts, The Jeweled Style : Poetry and Poetics in Late Antiquity, Ithaca and London 1989

Roberts $1989 \mathrm{~b}$

M. Roberts, The Use of Myth in Latin Epithalamia, in « TAPA » 119, 1989, 321-48

Rosati 1999

G. Rosati, Form in motion : weaving the text in the Metamorphoses, in Ovidian Transformations. Essays on Ovid's Metamorphoses and its Reception, edd. A. Barchiesi, Ph. Hardie, S. Hinds, Cambridge 1999, 240-53

Scheid-Svenbro 1994

J. Scheid-J. Svenbro, Le métier de Zeus. Mythe du tissage et du tissu dans le monde gréco-romain, Paris 1994

Schetter 1992

W. Schetter, Zur Publikation der 'Carmina minora' des Apollinaris Sidonius, in « Hermes » 120, 1992, 343-63

Uggeri 1966

G. Uggeri, Lussorio, Sidonio Apollinare e un'iconografia di Diogene e Laide, in « SIFC » 38, 1966, 246-55

Wheeler 1995

S.M. Wheeler, The Underworld Opening of Claudian's De Raptu Proserpinae, in « TAPA » 125, 1995, 113-34.

\section{NOTE}

1. Molto materiale in Scheid-Svenbro 1994.

2. Da vedere il bel lavoro di Gorni 1979 (che però nella storia della metafora sottovaluta l'importanza della letteratura latina, a vantaggio di quella romanza).

3. Rinvio su ciò a Rosati 1999.

4. Cfr. Barolini 1987.

5. Da vedere qui Barkan 1986, 5 ss.

6. Fra i migliori profili letterari di Sidonio cfr. Loyen 1943, Consolino 1974, Gualandri 1979, La Penna 1995. 
7. La composizione dell'epitalamio, anteriore a quella della lettera in prosa premessa alla prefazione che lo introduce (carm. 14), si data al 460 (cfr. Loyen 1960, XXXIII) o a pochi mesi prima (Schetter 1992, 353 n. 32; altre indicazioni in Kaufmann 1995, 336 e 281, con i dati prosopografici rispettivamente su Polemio e Araneola). Per un'informazione generale sul componimento rinvio a Ravenna 1990.

8. Dove si verifica anche l'allungamento, con conseguente spostamento dell'accento, della penultima, secondo un esito che avrà ampia fortuna nelle lingue romanze.

9. Almeno a stare al ThlL II 395, $37 \mathrm{~s}$.

10. Nota giustamente Pease 1955-58, ad loc. che questa è l'unica occorrenza certa di diminutivo al femminile : l'altra fornita dal ThlL II 395, 34, di Paul. Nol. carm. 16.118 araneolis, potrebbe anche essere al maschile (da accostare cioè a Culex 2 ut araneoli tenuem formavimus orsum).

11. Una funzione antonomastica simile a quella svolta nella caratterizzazione (peraltro opposta) di Clorinda nel Tasso: Gerusal. liber. 2.39 Costei gl'ingegni feminili e gli usi / tutti sprezzò sin da l'età più acerba ; / a i lavori d'Aracne, a l'ago, a i fusi / inchinar non degnò la man superba. Sull'esemplarità per la donna del lavoro del ragno cfr. sotto p. 00.

12. Due parole la cui «ressemblance est trop étroite» perché si possa ritenerle prive di connessione : cfr. Ernout-Meillet 1959, 42.

13. Ed è forse al confronto con Ovidio che allude la professione di modestia di $14.24 \mathrm{~s}$. nec, quod detonuit Camena maior, / nostram pauperiem silere cogas (per altre ipotesi cfr. Ravenna 1990 ad loc.). Un elenco di imitazioni ovidiane in Sidonio raccoglie il lavoro (anche poco informato) di Colton 2000 (136-79).

14. Cfr. Mesturini 1982, 263 s. (e 267 ss. su Apollinaris).

15. Cfr. anche, sull'ars e la ratio della tessitura del ragno, Plin. nat. 11.81 e in generale Capponi 1994, 129 ss.

16. A proposito di Polemio, Ravenna 1990, 17 n. 33 suggerisce giustamente che « una simmetrica interpretazione 'etimologizzante' potrebbe celarsi nell'atteggiamento inizialmente bellicoso di Atena ». Cfr. anche Roberts 1989b, 342.

17. Cfr. già Morelli 1910, 390.

18. Una tensione fra questi due mondi segnala Roberts $1989 \mathrm{~b}, 342$ s., che vede nelle rappresentazioni di Araneola una critica del rapporto gerarchico che essi presuppongono.

19. Sul gusto dei tessuti istoriati (e delle loro elaboratissime descrizioni) nella letteratura tardoantica è da vedere spec. Roberts 1989a, 111 ss.

20. L'identificazione (in base alla lezione patrio a 132 : cfr. Loyen 1960, ad loc.) del dio del mare mi pare sicura.

21. Sui particolari, e sulla tecnica ecfrastica, rinvio a Ravenna 1990, ad loc.

22. Sulla cui 'improprietà', almeno per il secondo, cfr. Ravenna 1990, ad loc.

23. Cui richiama la stessa definizione quod priscis inlustre toris (159); dove la menzione, dietro la metonimia toris, dell'oggetto concreto, del 'letto', funge da richiamo all'esempio più famoso, nella letteratura latina, di una coperta da letto istoriata con antiche vicende mitiche (vestis priscis hominum variata figuris, 64.50), quella offerta in dono in occasione di altre nozze, nozze semidivine, e descritta nel celebre carme catulliano.

24. La contraddittorietà con i quadri precedenti delle imprese di Giove è rilevata già da Purgold 1878, 113 (che la considera una 'conseguenza' dell'imitazione di Ovidio); mentre non vede il problema Ravenna 1990, 83 : «Tra i miti celebrati, i primi quattro riguardano la fedeltà e l'amore coniugale, gli altri tre (vv. 174-84) la seduzione, aspetti che l'epitalamio gradisce entrambi ». Morelli 1910, 392 accenna invece all'ipotesi che la sezione su Giove intenda « indicare la potenza d'amore che doma tutti gli dei ».

25. E proprio la ripresa al v. 176 di Dictynna, l'epiteto di Diana, è il segnale del rinvio all'episodio ovidiano, dove si legge (2.441) l'unica occorrenza del termine nel poema. 
26. Nella storia di questo nesso tracciata da Ravenna 1990 ad loc. va incluso, dietro il modello diretto di Claudiano (Cons. Olybr. 177), anche Stazio, Theb. 11.401 Maeoniis Argia modis ac pollice docto (relativo a un'altra tessitrice e sposa esemplare come Argia).

27. Così va inteso doctisonas... artes secondo Ravenna 1990 ad loc. : «Pallade non può essersi rivolta se non verso ciò che è pieno di dottrina, ossia il tempio dei filosofi. Solo questo può spiegare il fatto seguente, per cui Araneola, dovendo cambiare argomento, si sente autorizzata a rappresentare proprio un filosofo" (cfr. anche Roberts 1989b, 343). Diversamente Loyen 1960, che traduce: «quand Araneola, apercevant la Tritonide, vit qu'elle avait les yeux tournés vers elle et considérait avec un vif plaisir ses ouvrages si pleins de science » (ma così facendo annulla l'opposizione implicita tra ciò che Araneola sta tessendo e ciò che Minerva preferisce guardare : se le doctisonae artes sono quelle di Araneola, bisognerà piuttosto pensare alla prima parte del suo lavoro, e cioè i soggetti di amore coniugale).

28. Sull'aneddotica, diffusa nella 'filosofia popolare', relativa al rapporto fra Diogene e Laide informa bene Uggeri 1966.

29. Ravenna 1990, ad loc.

30. Uggeri 1966, 248.

31. Così Ravenna 1990, ad loc.

32. Già Morelli 1910, 388, rilevava questo aspetto a proposito delle notazioni di carattere programmatico della lettera iniziale : «ci mostra, in una specie di $\lambda \alpha \lambda_{1}$ ó sofistica, gli strumenti della sua professione ».

33. Inoltre l'immagine connessa del filare (delle Parche), che conclude il carme 15 così come iniziava il 14, incornicia tematicamente l'intero dittico.

34. Cfr. Bryson 1994, 266 ss.

35. E che è anche in grado di leggere : sulla dibattuta questione della sua conoscenza del greco da vedere le ponderate considerazioni di Loyen 1964, 83 ss.

36. Cfr. Purgold 1878, 109 ss.

37. Secondo Bryson 1994, 270 l'accostamento in sequenza dei quadri 2.27 (Nascita di Minerva) e 2.28 (Telai) implicherebbe anzi un richiamo al mito di Aracne.

38. Una ricchissima documentazione, anche bibliografica, fornisce ora G. Agosti nel comm. a Nonno, Par. 5.64 (in corso di stampa).

39. Cfr. von Albrecht 1989.

40. Basterà ricordare l'antica immagine della ragnatela tessuta sulle armi, un'immagine presente già in Bacchilide ma nota soprattutto da Theocr. 16.96-7 (« i ragni sulle armi distendano le loro tele sottili, e della guerra non rimanga neanche il nome »).

41. Così Gruzelier 1993, 257 ad loc.

42. Un'immagine che, secondo Gruzelier 1993, 143, coinciderebbe con la 'visione del mondo' dello stesso Claudiano.

43. Secondo von Albrecht 1999, il solo studioso che non si limita a un accenno generico all'episodio ovidiano di Aracne e Minerva, ma intuisce la rilevanza interpretativa del richiamo al modello, il peplo istoriato da Proserpina « becomes in a sense a palinode of Arachne's tapestry ; in fact, while in Ovid we have two competing views on world, society, and art, in Claudian there is only one » (321); e più avanti : « Proserpina's fabric in Claudian is an affirmative counterpart to Arachne's sacrilegious carpet in Ovid» (327). In realtà, io credo, l'immagine della ragnatela sul peplo abbandonato mostra che Claudiano non elide affatto la tensione presente nel testo tra le due opposte visioni del mondo.

44. Ma non credo, con Gruzelier ad loc., che vi si debba vedere l'intenzione « to create indignation over the destruction wrought on Proserpina's work ».

45. A Proserpina vittima dell'eros divino Aracne dedicava spazio nell'arazzo ovidiano (114 Deoida), ricordando l'accoppiamento con lei di Giove in forma di serpente (un'unione quindi addirittura incestuosa). 
46. Charlet 1991, XLIII s.

47. Se poi Claudiano condivida personalmente la fiduciosa ideologia provvidenzialistica rappresentata nel velo di Proserpina, o piuttosto se non l'animosità almeno la realistica sfiducia di Aracne, che lo farebbe guardare con ansia al sovvertimento che sta sconvolgendo il suo stesso mondo, è un questione su cui gli studiosi sono divisi: si vedano, in direzione opposta, rispettivamente Charlet 1991, XLVII e Kellner 1997, 286 s. Una posizione equilibrata in Wheeler 1995, spec. $132 \mathrm{~s}$.

48. L'unica menzione significativa, oltre al cit. verso di Giovenale, si legge in Manilio 4.136.

\section{AUTORE}

\section{GIANPIERO ROSATI}

Università di Udine 